\title{
A emergência de relações condicionais entre estímulos como resultado de treino de série de discriminações simples simultâneas ${ }^{1}$
}

\author{
The emergence of conditional relationships \\ among stimuli resulting from serial training \\ of simple, simultaneous discriminations
}

\author{
Maria Paula Soares MONTANS ${ }^{2}$ \\ Maria Amalia Pie Abib ANDERY²
}

\begin{abstract}
Resumo
O procedimento de emparelhamento de acordo com o modelo tem sido privilegiado para o estabelecimento de discriminações condicionais entre estímulos e para testar a emergência de classes de estímulos equivalentes. No entanto, outros procedimentos de treino discriminativo também têm sido efetivos para o estabelecimento destas classes. Este trabalho teve por objetivo estudar um procedimento de discriminação simples que, com base em discriminações simples já existentes, promoveria a emergência de relações condicionais entre estímulos. Participaram do estudo seis crianças (de seis e sete anos de idade) que trabalharam em computador. Depois de estabelecida uma discriminação simples entre dois estímulos, estes foram sobrepostos a novos estímulos, até a formação de nova discriminação. O mesmo procedimento foi repetido com outros estímulos. Depois de treino discriminativo no qual um participante apresentou apenas seis erros e os demais apresentaram três ou menos erros, testou-se a emergência de relações entre estímulos. Os testes revelaram que houve formação de classes de estímulos equivalentes para três participantes e sugeriram esta possibilidade para outros dois.
\end{abstract}

Unitermos: Controle de estímulos. Discriminação. Estímulo condicionado.

\begin{abstract}
A number ofstudies have shown the possibility of using series of simple discriminations and multiple reversals to establish equivalent stimulus classes. The current study aimed to test the possibility of establishing equivalence classes through a training procedure that established simple discriminations on the basis of previously established ones, without reversals. Participants consisted of 6 children, aged 6 and 7. After the initial discrimination training of a pair of stimuli, two new, simple discriminations were established, one at a time, using the superimposition of stimuli. Following training, conditional relations between stimuli were tested. Results showed that all participants learned the discriminations almost without error: one participant had 6 errors and all the others had three errors or fewer. Tests for the emergence of classes of equivalent stimuli were positive for 3 participants and suggested the possibility of such emergence for 2 other participants.
\end{abstract}

Uniterms: Stimulus control. Discrimination. Conditioned stimulus.

$\boldsymbol{\nabla \nabla v \nabla}$

1 Artigo elaborado a partir da dissertação de M.P.S. MONTANS, intitulada "Análise do Comportamento". Programa de Estudos Pós-Graduados em Psicologia Experimental: Análise do Comportamento. Pontifícia Universidade Católica de São Paulo, 2006. Apoio: Parcialmente financiado pela Coordenação de Aperfeiçoamento de Pessoal de Nível Superior.

2 Pontifícia Universidade Católica de São Paulo, Programa de Estudos Pós-Graduados em Psicologia Experimental: Análise do Comportamento. R. Monte Alegre, 1352,05014-002, São Paulo, SP, Brasil.Correspondência para/Correspondenceto:M.A. ANDERY.E-mails:<mandery@pucsp.br>; <mandery@uol.com.br>. 
Classes de estímulos foram definidas como conjuntos de estímulos (ou de propriedades, ou dimensões de estímulos) que afetam o responder de um organismo de uma determinada maneira (Skinner, 1959). Em 1993, de Rose afirmou que"três tipos de relações podem ocasionar a formação de classes de estímulos: similaridade física, relações arbitrárias mediadas por uma resposta comum e relações arbitrárias entre os estímulos" (p.287). Para De Rose (1993), como para Goldiamond (1966), a mediação de uma resposta comum com um conjunto de estímulos estabeleceria classes de estímulos funcionais, assim definidas por Sidman (1994): uma classe funcional é definida quando estímulos discriminativos diferentes num conjunto de contingências de três termos (discriminações simples) ocasionam a mesma resposta. Esse fenômeno tem sido referido pela literatura também como equivalência funcional (Sidman, 1986; Wirth \& Chase, 2002).

Outra possibilidade de formação de classes de estímulos envolveria o estabelecimento de relações arbitrárias entre os estímulos da classe, formando o que Sidman eTailby (1982) chamaram de classes de estímulos equivalentes. Essas classes seriam formadas após o treino de conjuntos de discriminações condicionais entre estímulos, que promoveriam a emergência de relações - não diretamente treinadas - de reflexividade, simetria e transitividade entre os estímulos.

Classes de estímulos equivalentes têm sido produzidas principalmente com o procedimento de pareamento com o modelo (matching to sample), pelo qual se estabelecem discriminações condicionais entre estímulos, testando-se então a emergência de relações entre estímulos com as propriedades definidoras das classes de estímulos equivalentes (Sidman, 1994), mas há também estudos que mostraram ser possível estabelecer classes de estímulos equivalentes com outros procedimentos, como os trabalhos de Svinicki, Kladder e Ponicki (1971), Zental e Hogan (1975), R.W. Mallot, K. Mallot eVaughan Jr. (1988), Debert (2003).

Vaughan Jr. (1988), em um estudo inovador para a época, propôs que contingências de três termos (discriminações simples) poderiam estabelecer classes de estímulos equivalentes. Trabalhou com pombos, estabelecendo uma série de discriminações simultâneas simples entre pares de estímulos (slides com imagens de árvores), seguida de reversões sucessivas de toda a série. Depois de algumas sessões com sucessivas reversões da série de slides (os slides estabelecidos como positivos tornavam-se negativos e vice-versa), a apresentação de apenas alguns slides da série era suficiente para que o responder dos sujeitos aos dois conjuntos se alterasse de acordo com a mudança nas contingências. $\mathrm{Na}$ interpretação de Vaughan Jr (1988), os slides que compunham os conjuntos positivo e negativo haviam se tornado membros de duas classes de estímulos, por meio de um processo que foi chamado pelo autor de partição, e estas seriam classes de estímulos equivalentes, uma vez que os sujeitos respondiam a um slide da classe como o faziam aos demais.

Sidman, Wynne, Maguire e Barnes (1989) criticaram a hipótese de Vaughan Jr. (1988) de que partição e relações de equivalência seriam matematicamente semelhantes, afirmando que ainda seria necessário testar se classes de estímulos funcionais e classes de estímulos equivalentes seriam comportamentalmente as mesmas. Para tanto, Sidman et al. (1989) conduziram um experimento com três rapazes com desenvolvimento atípico, no qual utilizaram o mesmo procedimento de produção de uma série de discriminações simples com reversões sucessivas para estabelecer classes de estímulos funcionais, mas adicionaram testes feitos via pareamento, de acordo com o modelo. Os estímulos apresentados no treino de discriminação simples foram números, para dois participantes, e letras gregas para o terceiro. Para todos os participantes a classe de estímulos designada A, com três estímulos (A1, A2 e A3), foi estabelecida como positiva, e a classe B, também com três estímulos (B1, B2 e B3), como negativa. Na fase 1, foi estabelecida a discriminação do primeiro par (A1xB1). Na fase 2, foi adicionado o segundo par (A2xB2) e, na fase 3, o terceiro par (A3×B3). Como no experimento de Vaughan Jr. (1988), após o treino as discriminações foram revertidas repetidamente. Para todos os participantes, a reversão das contingências para um par de estímulos tornou-se suficiente para que as respostas aos estímulos seguintes da mesma classe mudassem de acordo com as contingências em vigor e, assim, mostraram a emergência de classes funcionais, em decorrência de as reversões das discriminações simples estarem sob controle condicional exercido pela primeira tentativa, não reforçada ou reforçada. 
Encerrado este treino de discriminações simples, foram realizadas quatro fases de testes. Na primeira delas, verificou-se se os participantes relacionariam os estímulos das classes funcionais em um contexto de discriminação condicional sem reforçamento. Nas tentativas apresentava-se um estímulo-modelo e dois estímulos-comparação, cada um deles de uma das classes funcionais já estabelecidas. O desempenho dos três participantes foi considerado correto e os autores consideraram que havia evidência de emergência de relações condicionais não treinadas. Na segunda fase de testes, que demonstraria relações de equivalência entre os estímulos, testando simetria e transitividade, foram ensinadas relações condicionais entre um estímulo de cada classe (A1 e B1) e dois estímulos novos (A4 e B4, respectivamente) e testadas relações condicionais entre os estímulos A2 e B2 e os estímulos novos. Dois participantes tiveram resultados considerados positivos no teste. Já o desempenho do terceiro participante, que tivera dificuldades no teste anterior, foi marcado por muitos erros. Para testar a interação entre classes funcionais e classes de equivalência foram ensinadas aos dois participantes com resultados positivos nos testes anteriores discriminações condicionais entre os estímulos A4 e B4 e mais dois novos estímulos (A5 e B5), e testadas as relações entre estes e os estímulos A2 e B2. Ambos os participantes tiveram resultados considerados positivos. Por fim, para confirmar a inclusão dos novos estímulos nas classes funcionais estabelecidas por discriminação simples, os dois novos pares de estímulos (A4/B4 e A5/B5) foram acrescidos aos três pares originais, em tentativas reforçadas de discriminação simultânea simples. Os poucos erros sugeriram que os novos estímulos tinham realmente passado a fazer parte das classes funcionais.

Apesar dos resultados positivos com dois participantes, Sidman et al. (1989) recusaram a hipótese de Vaughan Jr. (1988) de que classes de estímulo equivalentes e classes de estímulos funcionais representariam o mesmo processo comportamental, já que o desempenho de um dos participantes mostrava a formação de classes de estímulos funcionais, mas não a formação de classes de estímulos equivalentes. Em 1994, Sidman reviu sua afirmação anterior, afirmando que os desempenhos dos dois participantes que indicavam a formação de classes de estímulos equivalentes poderiam ser tomados como suporte da posição de Vaughan Jr. (1988) de que "partição"implicaria relação de equivalência entre estímulos e que classes de estímulos equivalentes e funcionais seriam dois lados de uma mesma moeda. Nesses casos, afirmou Sidman, os estímulos de cada classe controlavam a mesma resposta, a mesma consequência da resposta, e as classes de estímulos poderiam ser chamadas de "classes de contingência", estabelecidas pelo procedimento de reversões sucessivas, envolvendo no mínimo dois subconjuntos de estímulos, uma única resposta definida e um único reforçador definido (Sidman, 2000). A discussão sobre o papel das discriminações condicionais na formação de classes de estímulos equivalentes foi retomada por Sidman (1994), que concluiu seu artigo afirmando que o procedimento de reversões sucessivas que produziu discriminações condicionais aparentemente emergentes poderia tê-las ensinado diretamente: o procedimento de reversões repetidas poderia envolver discriminação condicional, ou seja, um estímulo correlacionado com reforçamento (ou não reforçamento) em uma tentativa poderia ter a função de estímulo-modelo na tentativa seguinte.

Independentemente dessa discussão, o procedimento de reversões repetidas tem sido utilizado para produzir classes de estímulos funcionais. Classes de estímulos funcionais têm sido produzidas também com contingências de três termos com um procedimento de discriminação simples com respostas definidas (Jitsumori, Siemann, Lehr \& Delius, 2002; Kastak, Schusterman \& Schusterman, 2001; Tomonaga, 1999).

Wirth e Chase (2002), por exemplo, utilizaram um procedimento que estabeleceu classes de estímulos funcionais com oito estudantes universitários, com uma tarefa na qual eles foram treinados a dizer três palavras sem sentido na presença de três conjuntos de três estímulos. Utilizando computador que reconhecia as vozes dos participantes, três sílabas eram apresentadas na tela aos participantes que as liam. Na fase 2, um de nove estímulos arbitrários era apresentado na tela do computador e os participantes deveriam dizer uma das três sílabas diante de cada um deles. Os estímulos visuais da classe 1 foram denominados A1, B1 ou C1, os da classe 2 foram denominados A2, B2, C2 e os da classe 3 foram chamados de A3, B3, ou C3. As respostas de dizer as sílabas foram relacionadas a números correspon- 
dentes às classes de estímulos que as evocariam: R1, R2, ou R3. Encerrado o treino discriminativo, os participantes emitiam a resposta $\mathrm{R} 1$ diante dos três estímulos visuais designados como classe 1 (A1, B1 e C1), o mesmo ocorrendo com as respostas R2 e R3. Fez-se então um novo treino de "reconhecimento da fala" com novas sílabas ( $R 4$, R5 e R6) e um novo treino no qual estas respostas foram reforçadas na presença de $\mathrm{A} 1, \mathrm{~A} 2$ e $\mathrm{A} 3$, respectivamente. Para testar a formação de classes de estímulos funcionais, os estímulos A, B e C das três classes foram apresentados para verificar a ocorrência de"transferência de função" e de formação de classes de estímulos funcionais. Os resultados iniciais foram positivos apenas para um participante, mas o treino adicional produziu desempenho considerado positivo para a formação de classes de estímulos funcionalmente equivalentes nos demais.

Ainda como exemplo de estudos que testaram a emergência de classes de estímulos equivalentes sem usar exclusivamente procedimento de treino de pareamento de acordo com o modelo, Tyndall, Roche e James (2004) conduziram um estudo com 57 estudantes para testar se a função comportamental do estímulo neste caso, função discriminativa - dificultaria ou não a formação de classes de equivalência envolvendo tais estímulos. Um procedimento de discriminação simples sem reversões foi usado para estabelecer seis sílabas sem sentido como estímulos discriminativos (A1, B1, C1 e A3, B3, C3) e outras seis como estímulos delta (A2, $B 2, C 2$ e A4, B4, C4). No treino discriminativo foram apresentados pares de estímulos pertencentes à presumida classe $1\left(\mathrm{~S}^{+}\right)$e classe $2\left(\mathrm{~S}^{-}\right)$, e pares formados por $\mathrm{S}^{+}$ da classe 3 e $S$ da classe 4 . Todos os participantes atingiram rapidamente o critério, estabelecendo-se duas classes funcionais: A1, B1, C1, A3, B3 e C3 como $\mathrm{S}^{+}$, e A2, $B 2, C 2, A 4, B 4$ e C4 como S- Na segunda fase (de treino de pareamento de acordo com o modelo) os participantes foram divididos em quatro grupos e foram expostos a uma entre quatro condições experimentais. A diferença entre as condições consistia nos estímulos utilizados como modelo e comparação. No primeiro grupo de participantes (condição $S^{+}$) foram estímulos-modelo e comparação de todos os $\mathrm{S}^{+}$, e foram formadas duas classes de estímulos: A1B1C1 e A3B3C3. No segundo grupo (condição $S^{+} / S^{-}$) foram formadas as classes A1B1C1/A4B4C4. No terceiro grupo (condição $\mathrm{S}^{+} / \mathrm{S}^{-}$misturados), os $\mathrm{S}^{+}$e os $\mathrm{S}^{-}$foram aleatoriamente distribuídos como modelo e comparação, formando as classes A1B1C4/A4B4C1. Finalmente, no quarto grupo (condição S) todos os estímulos eram S-, formando-se as classes A2B2C2 e A4B4C4. Um grupo controle, com participantes que não passaram pelo treino de discriminação simples, foi exposto à condição $\mathrm{S}^{+}$. Todos os participantes de todos os grupos experimentais e do grupo controle atingiram o critério de desempenho no treino de pareamento de acordo com o modelo; os participantes do grupo $\mathrm{S}^{+} / \mathrm{S}^{-}$misturados atingiram o critério com menos treino. Nos testes de equivalência que verificaram a emergência das relações $\mathrm{A}-\mathrm{C}$ e C-A, 44 dos cinquenta participantes formaram classes de equivalência. Dois participantes do grupo $S^{+}$, três do grupo $\mathrm{S}^{+} / \mathrm{S}^{-}$misturados e um participante do grupo controle tiveram desempenhos que não indicaram relações emergentes. Nos grupos $\mathrm{S}^{+} / \mathrm{S}^{-}$e $\mathrm{S}^{-}$os dez participantes tiveram desempenhos que indicaram simetria, transitividade e equivalência entre os estímulos. Ressalte-se que Tyndall et al. (2004) discutem a impossibilidade de afirmar com certeza se os participantes da condição $\mathrm{S}^{+} / \mathrm{S}$ formaram classes de estímulos equivalentes ou se simplesmente separaram os estímulos positivos e negativos em grupos funcionais por escolherem os estímulos-comparação da mesma classe do modelo.

Apesar de dúvidas como esta, parece que outros procedimentos, além de extensos treinos de pareamento de acordo com o modelo, promovem o estabelecimento de classes de estímulos equivalentes e/ou de classes de estímulos funcionalmente equivalentes, como aqueles testados porVaughan Jr. (1988) e por Sidman et al. (1989). Do mesmo modo, estudos como os de Wirth e Chase (2002) e Tyndall et al. (2004) indicam que há relações entre treino discriminativo e formação de classes de estímulos equivalentes ou funcionais que merecem investigação. Esses estudos sugerem que há interações entre o estabelecimento de discriminações simples envolvendo séries de estímulos e a formação de classes de estímulos funcionais e, aparentemente, em certos casos, também de classes de estímulos equivalentes. Esta constatação torna relevante a investigação de procedimentos de treino de discriminação simples que poderiam ser promotores ou facilitadores da formação de classes de estímulos equivalentes.

Um procedimento que parece promissor foi desenvolvido por Touchette (1971), que treinou novas 
discriminações usando algumas já estabelecidas, em um procedimento que permitia identificar o momento no qual ocorria a transferência de controle de estímulos de um conjunto de propriedades de estímulo (a antiga discriminação) para um segundo conjunto (a discriminação nova). Estabelecida uma discriminação simples entre as cores vermelho $\left(\mathrm{S}^{+}\right)$e branco $\left(\mathrm{S}^{-}\right)$, os participantes do estudo deTouchette - rapazes com desenvolvimento atípico - passaram por novo treino discriminativo entre duas formas: a letra E deitada com as pernas apontadas para baixo $\left(\mathrm{S}^{+}\right)$e deitada e para cima $\left(\mathrm{S}^{-}\right)$. Na primeira tentativa de cada série de 32 tentativas, o S+ era apresentado sobreposto à cor vermelha. Uma resposta correta (tocar no estímulo $\mathrm{S}^{+}$) afetava a tentativa seguinte, adiando o aparecimento da cor vermelha em 0,5 segundo. Foram consideradas corretas as respostas emitidas antes ou depois do aparecimento da cor vermelha sobre o $\mathrm{S}^{+}$. Respostas incorretas finalizavam uma tentativa e reduziam o tempo decorrido entre o início da tentativa e o aparecimento da cor vermelha em 0,5 segundo na tentativa subsequente. Encerrado o treino discriminativo, os participantes foram submetidos a sucessivas reversões, utilizando-se o mesmo procedimento. Os participantes quase não cometeram erros desde as primeiras tentativas do primeiro treino de discriminação, e depois de algumas reversões passaram a responder à dimensão forma antes que a cor fosse apresentada.

Bonito (2005), com base em Touchette (1971), testou um procedimento que pudesse ser útil no estudo do processo envolvido na discriminação. Participaram do estudo seis crianças de 4 e 5 anos. Depois de um pré-treino de familiarização com a tarefa e de manuseio do mouse em um computador, iniciou-se um treino discriminativo por tentativas nas quais eram apresentados dois quadrados - um amarelo e um azul - na tela do computador. Respostas no quadrado convencionado como $\mathrm{S}^{+}$eram sinalizadas como acerto e respostas no quadrado convencionado como S- não tinham consequência programada. Nas três fases seguintes, foram treinadas três novas discriminações (entre símbolos arbitrários da fonte Wingdings): um par de estímulos em cada fase. Na primeira tentativa de cada fase, os estímulos convencionados como $\mathrm{S}^{+}$e $\mathrm{S}^{-}$eram sobrepostos aos $\mathrm{S}^{+}$e $\mathrm{S}^{-}$estabelecidos como tal na primeira fase (as cores amarelo e azul, respectivamente). A resposta de escolha do $\mathrm{S}^{+}$tinha consequência de acerto e afetava a tentativa seguinte, postergando a sobreposição das cores sobre os estímulos em cinco segundos para alguns participantes, ou dez segundos para outros. Respostas incorretas encerravam a tentativa e a mesma configuração de estímulos era apresentada na tentativa seguinte, com a sobreposição das cores aos novos estímulos adiantada em cinco segundos. Em cada fase, foram apresentados blocos de 12 tentativas, nas quais as posições dos estímulos na tela mudavam. Atingido o critério de discriminação em cada uma dessas fases, foram apresentadas seis tentativas-teste: em três delas, o estímulo estabelecido como $S^{+}$na respectiva fase era apresentado simultaneamente com um estímulo novo - diferente dos anteriores - e nas outras três o S- era apresentado com um outro estímulo novo. As tentativas-teste foram apresentadas em ordem aleatória e em extinção. Os participantes aprenderam as discriminações quase sem erro e os resultados dos testes sugeriram, para quatro dos seis participantes, controle do responder tanto pela escolha do $\mathrm{S}^{+}$quanto pela exclusão do S, evidenciado pela escolha do $\mathrm{S}^{+}$quando este era apresentado com um estímulo novo e do estímulo novo quando este apresentado com o $\mathrm{S}$ :

Os estudos de Touchette (1971) e Bonito (2005) envolveram treino discriminativo em que pares de estímulos foram tornados $\mathrm{S}^{+}$e $\mathrm{S}^{-}$, a partir de discriminações já estabelecidas, com muito poucas tentativas e praticamente sem erros. Seus resultados sugeriram a possibilidade de que um treino que envolvesse apenas discriminações simples, sem reversão, construídas a partir de discriminações já estabelecidas, poderia produzir classes de estímulos equivalentes, testadas com procedimentos de pareamento de acordo com o modelo. Esta possibilidade foi testada no trabalho aqui descrito.

\section{Método}

\section{Participantes}

Participaram do estudo seis crianças com 6 e 7 anos, de ambos os sexos. Os pais assinaram Termo de Consentimento Livre e Esclarecido e o projeto foi aprovado pelo Comitê de Ética da instituição de filiação dos autores. 
Equipamento

Foi utilizado um software que controlava a apresentação de estímulos e os registros necessários, desenvolvido por Renato Inamine, operado em um computador do tipo laptop com mouse acoplado.

Brinquedos foram usados como prêmios para os participantes; ao final de cada etapa ou fase, as crianças eram convidadas a trocar seus pontos por brinquedos de sua escolha.

\section{Coleta de dados}

As sessões ocorreram em uma sala de aproximadamente $20 \mathrm{~m}^{2}$ mobiliada com mesa e cadeiras. $\mathrm{O}$ participante e o experimentador (que permanecia na sala durante a sessão) sentavam-se lado a lado nas cadeiras em frente à mesa, na qual estava o computador.

\section{Procedimento}

O procedimento envolveu pré-treino e quatro fases: 1) treino discriminativo dos conjuntos de estímulos denominados $\mathrm{A}, \mathrm{B}$ e $\mathrm{C}$; 2 ) treino discriminativo dos três conjuntos com $100 \%$ de reforço e $50 \%$ de reforço; 3) treino de discriminação condicional; 4) teste de relações emergentes.

\section{Pré-treino do uso do equipamento}

Foi feito um treino de uso do equipamento para familiarizar a criança com o computador e o manuseio do mouse. Para tanto, o participante era convidado a clicar com o mouse sobre um de dois quadrados (de $6 \mathrm{~cm}$ de lado) existentes na tela do computador. A cada clique seguia-se a apresentação de balões que subiam pela tela e de um som. Esse treino era encerrado quando o participante clicava com o mouse sem hesitação sobre um dos quadrados, em três tentativas consecutivas.

Fase 1: Treino discriminativo dos conjuntos de estímulos A, Be C: no início da fase, o participante recebia instruções gerais sobre o procedimento, especialmente de troca de pontos por brinquedos.

No treino discriminativo foram estabelecidas, separadamente, discriminações de três pares de estímulos, denominados conjuntos $\mathrm{A}, \mathrm{B}$ e C. O conjunto $\mathrm{A}$ foi formado pelos estímulos vermelho (A1) e azul (A2), e os conjuntos B (B1 e B2) e C ( 1 1 e C2) por dois estímulos arbitrários (da fonte Wingdings). Para metade dos participantes, os $\mathrm{S}^{+}$foram os estímulos designados como classe 1 e os $\mathrm{S}$ como classe 2, e para a outra metade dos participantes foi estabelecido o inverso.

As tentativas foram organizadas em blocos (de 12 tentativas, quando não havia erro) que envolviam a apresentação do par de estímulos em dois quadrados de $6 \mathrm{~cm}$ de lado, em 12 posições dos estímulos na tela.

Uma tentativa era iniciada com a apresentação na tela de dois estímulos de um conjunto e com um som (com duração de 1s), que sinalizava o início da tentativa. O participante usava o mouse para escolher um dos estímulos, clicando sobre ele.

Toda resposta de clicar sobre o estímulo pertencente à classe estabelecida como positiva era considerada correta e tinha como consequência a apresentação de um entre dois sons, de balões coloridos que subiam pela tela e do acréscimo de pontos, representado pelo crescimento de uma árvore colorida localizada na parte direita da tela. Em seguida, havia um intervalo entre tentativas (IET) de 4s, durante o qual a tela ficava branca, após o que uma nova tentativa era iniciada. Uma resposta de clicar sobre o estímulo pertencente à classe estabelecida como negativa era considerada incorreta e tinha como consequência o desaparecimento dos estímulos, seguido do IET e da mesma configuração de estímulos na tentativa seguinte. O treino discriminativo de um par de estímulos era encerrado quando o participante atingia o critério de 90\% de acertos em um bloco. Se, ao final de um bloco, o critério não tivesse sido atingido, um novo bloco era apresentado.

Encerrado o treino do conjunto $A$ (amarelo $x$ azul) iniciou-se o treino discriminativo dos conjuntos $B$ e $C$, sucessivamente. As mesmas contingências gerais foram mantidas no treino discriminativo dos estímulos B e C, com uma importante diferença: na primeira tentativa do treino do novo par de estímulos B (B1×B2) - e, depois, do par C (C1XC2) - os estímulos apareciam com as cores (estímulos A) sobrepostas a eles; ou seja, o estímulo que se tornaria $\mathrm{S}^{+}$tinha sobreposta a cor estabelecida como S+, e o estímulo estabelecido como S; a cor estabelecida como S. 
Respostas corretas tinham as mesmas consequências especificadas acima e modificavam a tentativa seguinte, acrescentando $5 \mathrm{~s}$ - contados desde o início da tentativa - antes do aparecimento das cores sobre os estímulos. Se o participante clicasse sobre o $\mathrm{S}^{+}$antes da sobreposição, a resposta também era considerada correta e a tentativa era encerrada antes que ocorresse a sobreposição. Respostas incorretas afetavam a tentativa seguinte diminuindo em cinco segundos o tempo necessário para o aparecimento das cores sobre os estímulos.

Fase 2: Treino discriminativo dos três conjuntos mixados: nesta fase, foram apresentados blocos com quatro tentativas de cada um dos três pares de estímulos treinados, em ordem aleatória e sem sobreposição de cor. Todas as tentativas com respostas consideradas corretas foram reforçadas. Atingidos $90 \%$ de acertos no bloco, um novo bloco mixado de 12 tentativas era apresentado, no qual apenas 50\% das tentativas consideradas corretas eram reforçadas. Foi critério de encerramento da fase pelo menos $90 \%$ de acertos em cada bloco.

Fase 3: Treino de pareamento de acordo com o modelo: primeiramente foi feito um pré-treino de identidade: o participante recebia instruções que descreviam a tela e, então, apresentava-se uma tentativa na qual um estímulo-modelo - um dentre dois quadrados coloridos - era apresentado. Quando o participante clicava sobre o estímulo, dois estímulos-comparação (dois quadrados coloridos, um deles da mesma cor do estímulo-modelo) eram apresentados. Respostas ao estímulo-comparação da mesma cor que o estímulo-modelo eram consequenciadas como acerto. Quando o participante acertava duas tentativas consecutivas, era iniciado o treino.

Neste treino, um novo par de estímulos denominados D1 e D2 (da fonte Wingdings) foi relacionado aos estímulos A1 e A2, respectivamente, mediante reforçamento diferencial: cada tentativa era iniciada com a apresentação de um estímulo do conjunto A (A1 ou A2). Após um clique do mouse sobre esse estímulo, os dois estímulos do conjunto Deram apresentados como estímulos-comparação em quaisquer duas de quatro posições ao redor do quadrado central. Quando o estímulo-modelo era A1, a resposta de seleção do estímulo-comparação D1 (A1D1) era considerada correta, e quando o estímulo-modelo era A2, a seleção de D2 (A2D2) era considerada correta. Todas as respostas corretas e incorretas eram seguidas pelas mesmas consequências do treino discriminativo. Quando o participante atingia 90\% de acertos no bloco de 12 tentativas, o treino de discriminação condicional era encerrado.

Fase 4: Testes de relações emergentes entre estímulos: esta fase teve por objetivo testar a emergência de relações condicionais entre os estímulos que fizeram parte do treino discriminativo de pares de estímulos ( $A$, $\mathrm{B} \mathrm{e}$ C) e os estímulos-modelo do treino de pareamento de acordo com o modelo, que estabeleceu a relação AD. O mesmo procedimento de apresentação de estímulos da fase anterior foi utilizado; no entanto, não havia consequência diferencial programada para as respostas nas tentativas de teste.

Nesta fase, foram apresentados três blocos de 21 tentativas em ordem aleatória. Cada bloco era composto de 15 tentativas de teste (sem consequências diferenciais) e seis tentativas de treino (com as mesmas consequências diferenciais das fases anteriores).

As 15 tentativas-teste foram distribuídas em 14 tentativas de pareamento de acordo com o modelo (discriminação condicional) e uma tentativa de discriminação simples. As 14 tentativas de teste de discriminação condicional envolveram: oito tentativas de teste das relações DB (D1B1 e D2B2), BD (B1D1 e B2D2), CD (C1D1 e C2D2), e DC (D1C1 e D2C2) e seis tentativas de teste em que os estímulos-cores (A2 ou A1) estabelecidos como $\mathrm{S}$ - foram apresentados como estímulosmodelo ou estímulo-comparação correto, relacionados com os demais estímulos dos treinos discriminativos (B2, C2, ou B1, A1); ou seja, foram testadas as relações $A 2 B 2, A 2 C 2, B 2 A 2, B 2 C 2, C 2 A 2$ e C2B2 (ou A1B1, A1C1, $B 1 A 1, B 1 C 1, C 1 B 1$ e C1A1 para metade dos participantes). Na tentativa-teste de discriminação simples (15a tentativa de teste), os dois estímulos do conjunto D (um deles pareado com um $\mathrm{S}^{+}$e outro com um $\mathrm{S}^{-}$no treino discriminativo) eram apresentados em dois quadrados, lado a lado.

As seis tentativas-treino, inseridas para intercalar tentativas reforçadas com as tentativas-teste, eram tentativas de discriminação simples iguais às tentativas de treino discriminativo, sem a sobreposição de cor. Cada par de estímulos foi apresentado duas vezes. 


\section{Resultados}

Na Figura 1 estão representados os resultados do treino discriminativo, por bloco de treino. Cada painel mostra o desempenho de um participante. No eixo $Y$ estão representadas as 12 tentativas de cada bloco de treino, e as colunas representam os acertos em cada um dos treinos discriminativos; nas colunas brancas estão representadas as tentativas em que houve sobreposição das cores sobre os estímulos nos treinos de discriminação simples. As colunas pretas mostram os acertos nos treinos A1 XA2, B1 xB2 e C1xC2; as colunas cinza, o treino mixado e a última coluna (cinza escuro) representam o treino de discriminação condicional $A D$ (que precedeu os testes).

A análise da Figura 1 indica que os participantes cometeram poucos erros. Desconsiderando-se os erros que ocorreram na primeira tentativa dos treinos discriminativos novos (e que foram considerados na construção da Figura 1), houve apenas três erros no treino da primeira (discriminação A1XA2) do participante N, e dois erros no treino de discriminação C1xC2 do participante Y. No treino discriminativo entre os estímulos C, os dois erros cometidos pelo participante $Y$ ocorreram em tentativas nas quais ele respondeu antes da sobreposição de cores (estímulos A). No treino mixado (em que houve tentativas com os estímulos A, B, ou C), apenas o participante $Y$ cometeu um erro em cada um dos dois blocos apresentados, um resultado que, em geral, indica a manutenção do controle discriminativo anteriormente estabelecido.

A Figura 1 indica também que foram necessárias poucas sobreposições de cores (estímulos A) antes que os participantes passassem a responder discriminativamente diante dos estímulos B e C: de duas a quatro sobreposições em cada treino. Em todos os casos, não houve erros nas tentativas em que houve sobreposição de cores, indicando que esta dimensão de estímulos cor - controlava discriminativamente o responder, e que a sobreposição sobre estímulos desconhecidos foi suficiente para que se estabelecesse controle discriminativo pelos novos estímulos.

O treino de discriminação condicional (relação AD) também foi encerrado com relativamente poucas tentativas; o participante $Y$ foi o único a ser submetido a dois blocos de 12 tentativas para que seu desempenho atingisse o critério estabelecido para encerramento do treino.

Os resultados nos testes de relações entre estímulos estão representados na Figura 2. Cada painel mostra o desempenho de um participante nos testes. A porcentagem de acertos em cada relação testada é representada pela altura das barras. Nas barras pretas estão apresentados os desempenhos nos testes das relações condicionais $A C, A B, B C, B A, C A, C B$, nas quais os estímulos-modelo sempre foram $S$; e nas barras brancas figuram as relações $B D, D B, C D, D C$. Na última barra (cinza) representa-se a porcentagem de acertos dos participantes quando os estímulos D1 e D2 foram apresentados.

O exame da Figura 2 indica que, para três dos seis participantes (A, LU e V), o treino discriminativo foi suficiente para estabelecer duas classes de estímulos equivalentes. Os testes BD, DB, CD, DC envolveram relações entre estímulos que não haviam sido pareados em nenhuma tentativa anterior e podem ser considerados como testes de transitividade e equivalência, uma vez que se treinou apenas a relação AD e parearam-se apenas os estímulos A-B e A-C. Nesses testes, os participantes $A$, $L U$ e $V$ responderam com apenas um ou nenhum erro. Os testes das relações $A C, A B, B C, B A, C A$ e CB envolveram pares de estímulos que foram apresentados simultaneamente no treino e pares que não o foram. Seriam semelhantes a testes de simetria ( $A B, B A$, $A C, C A)$ e de transitividade (CB, BC); no entanto, como apenas os estímulos estabelecidos como estímulos delta $\left(\mathrm{S}^{-}\right.$) no treino discriminativo foram apresentados como estímulos-modelo, para acertar os participantes teriam que responder estritamente sob controle da relação entre estímulos, ainda que essa relação envolvesse como estímulo-modelo e como estímulo-comparação dois estímulos estabelecidos como S. Embora tenha havido mais erros nesse teste em comparação com o anterior, como indicado na Figura 2, os participantes A, LU e V tiveram desempenhos que sugerem, mais uma vez, o estabelecimento de classes de estímulos equivalentes.

O último ponto de cada painel (Figura 2) representa o percentual de acertos de cada participante nas tentativas em que os estímulos $D$ foram apresentados isolada (sem qualquer outro estímulo) e simultaneamente. Essas tentativas eram semelhantes a tentativas de 

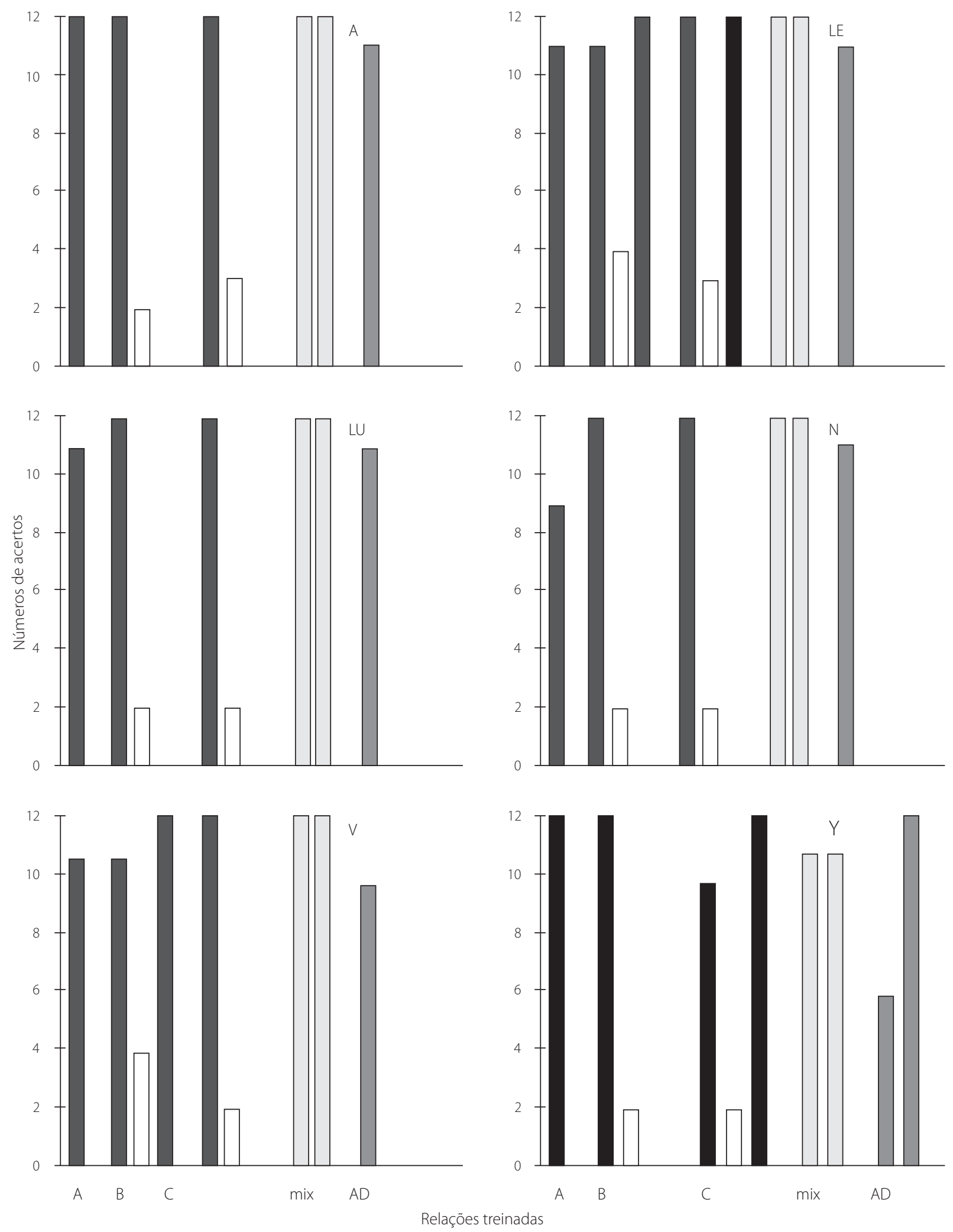

Figura 1. Resultados do treino discriminativo (número de acertos) por participante.

Nota: Cada painel representa o desempenho de um participante. As colunas pretas indicam desempenho nos treinos iniciais de discriminação simples e as colunas brancas representam o número de tentativas em que a resposta ocorreu apenas depois da sobreposição da cor. Nas colunas cinza, apresenta-se o desempenho nos blocos mixados e, na coluna cinza, o desempenho do participante no bloco de treino da discriminação condicional AD. 
discriminação simples, e com elas pre-tendia-se testar se cada um desses estímulos que participaram do treino de discriminação condicional AD assumiu função discriminativa (de $\mathrm{S}^{+}$ou de $\mathrm{S}^{-}$). Nessas tentativas, apenas o participante $V$ apresentou $100 \%$ de acertos; os participantes LU, LE, N e Y tiveram 67\% de acertos, e o participante A acertou apenas uma das três tentativas. O exame da sequência de tentativas revelou que, em todas as tentativas de discriminação simples, o participante A respondeu escolhendo um estímulo da
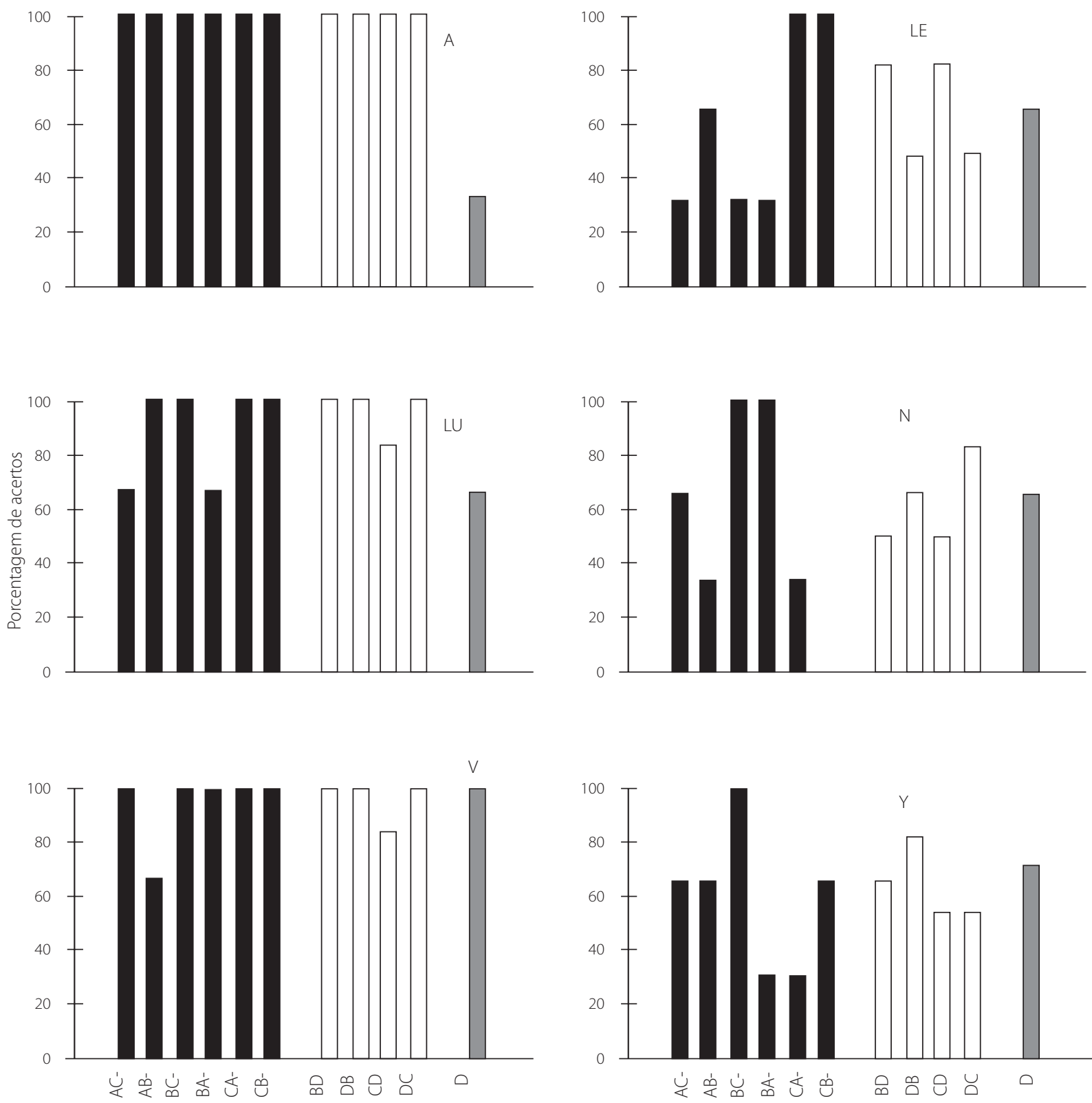

Relações testadas

Figura 2. Resultados dos testes de relações emergentes por participante.

Nota: Cada painel representa o desempenho de um participante. As colunas pretas indicam desempenhos nos testes que envolveram os estímulos do treino de discriminação simples (estímulos A, B, C). Nas colunas brancas estão representadas relações envolvendo estímulos que participaram dos treinos de discriminação simples e da discriminação condicional AD. Na coluna cinza, representa-se o desempenho nas tentativas com formato de discriminação simples com os estímulos D (D1xD2). 
mesma classe funcional da tentativa anterior, indicando que a sequência das tentativas controlou seu responder. Para todos os participantes, o teste envolveu tentativas com formato de discriminação simples, em que havia reforçamento diferencial (com os estímulos A1XA2, B1XBe e (1xC2), e tentativas com o mesmo formato de discriminação simples, sem reforçamento programado (com os estímulos D1xD2). A extinção do responder pode ter induzido variabilidade.

Já os desempenhos nos testes dos participantes N, LE e Y (Figura 2), não sugerem a formação de classes de estímulos equivalentes, ainda que no treino discriminativo esses participantes não tivessem um desempenho obviamente diferente dos demais. Nos testes AC, $A B, B C, B A, C A$ e $C B$ os participantes N, LE e $Y$ apresentaram muitos erros, sugerindo a possibilidade de controle por exclusão do $S$; uma vez que esses testes envolveram sempre estímulos-modelo estabelecidos como $S^{-}$no treino ou estímulos associados a $\mathrm{S}$.

Uma vez que nos testes de discriminação condicional BD, DB, CD e DC houve tentativas de teste de relações entre estímulos que envolviam estímulos-modelo estabelecidos como S- (e, portanto, também estímulos-comparação estabelecidos como S-) e tentativas em que os estímulos-modelo foram estabecidos como S+, construiu-se a Figura 3. Nela, está representado o número de tentativas corretas em cada relação testada, separando-se aquelas que envolviam a escolha de um estímulo-comparação estabelecido como S- (ou associado a um $\mathrm{S}^{-}$) e as que envolviam escolha de $\mathrm{S}^{+}$.

Lembrando que cada uma das relações (BD, DB, CD e DC) envolvendo $S^{-}$e envolvendo apenas $\mathrm{S}^{+}$foi testada três vezes (em três blocos), o exame da Figura 3 mostra que o participante LE acertou todas as tentativas que não envolviam a escolha de um S ou de um estímulo associado a $S$, e muito poucas tentativas (apenas três de 12) que envolviam escolher um S- ou um estímulo associado a um $\mathrm{S}^{-}$no treino discriminativo. Embora o participante $\mathrm{N}$ tenha apresentado quatro erros nas tentativas que não envolviam escolha do S- (relações DB, BD e DC), houve claramente menos acertos nas tentativas em que a resposta correta envolvia escolher um estímulo delta ou um estímulo que havia sido associado aos estímulos delta (apenas um acerto em 12 tentativas). Esses desempenhos dos particpantes LE e N sugerem

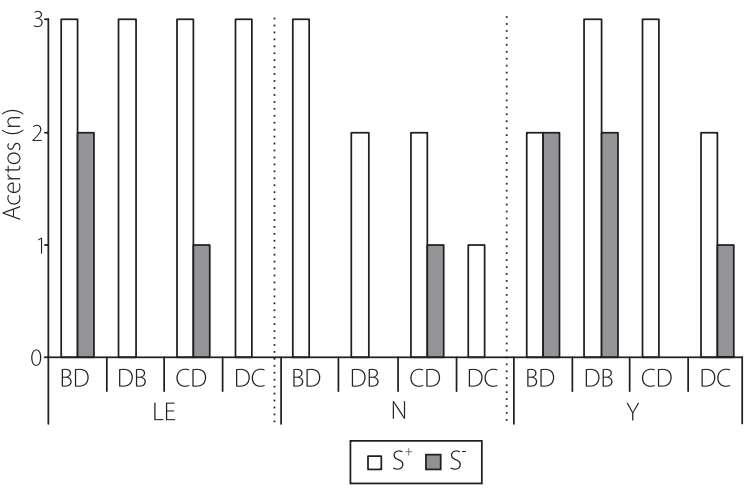

Relações testadas por participante

Figura 3. Desempenho dos participantes $L E, N$ e $Y$ nos testes de discriminação condicional envolvendo os estímulos D.

Nota: As barras brancas representam os acertos quando o estímulo-modelo ou estímulo-comparação correto era um estímulo antes estabelecido como $\mathrm{S}^{+}$. As barras cinza representam o número de acertos quando o estímulo-modelo ou estímulo-comparação foi estabelecido como S.

que é possível que tenham sido estabelecidas, também com esses participantes, classes de estímulos equivalentes; no entanto, essas classes também seriam claramente classes funcionais, e os participantes comportaram-se nos testes de modo a evitar a escolha dos estímulos com função de $S$.

\section{Discussão}

Este estudo mostrou que o procedimento empre-gado para estabelecimento de discriminação simples foi eficaz: os seis participantes aprenderam as discrimi-nações dos estímulos dos conjuntos A, e especialmente dos conjuntos B e C, sem erro ou quase sem erro, com pouco treino e relativamente poucas sobreposições de estímulos, uma vez que o participante que mais vezes foi exposto a tentativas em que dois estímulos eram sobrepostos foi o PY (sete tentativas).

O estabelecimento de discriminações simples também parece ter sido condição facilitadora do estabelecimento de discriminação condicional, uma vez que, de todos os participantes, apenas um (mais uma vez o PY) foi exposto a dois blocos de 12 tentativas de treino de discriminação condicional AD. 
Por último, o treino discriminativo foi suficiente para promover a formação de duas classes de estímulos equivalentes compostas dos estímulos envolvidos no treino, cada uma delas com os estímulos associados com a função de $\mathrm{S}^{+}$ou de $\mathrm{S}$ para uma parcela dos participantes.

Hoje, reconhece-se que outros procedimentos de treino, que não envolvem necessariamente pareamento de acordo com o modelo, podem ser suficientes para a construção de classes de estímulos equivalentes (Debert, 2003; Mallot et al., 1971; Vaughan Jr., 1988; Zental \& Hogan, 1975). Destaca-se entre esses procedimentos o treino de séries de discriminações simples com sucessivas reversões de toda a série (Jitsumori et al., 2002; Kastak et al., 2001; Sidman et al., 1989; Tomonaga, 1999; Vaughan Jr., 1988). No estudo aqui descrito também foi usado o treino de séries de discriminações, no entanto, não foram feitas reversões, que têm sido consideradas característica essencial do procedimento de treino que envolve séries de discriminações simples e a obtenção de classes de estímulos equivalentes (Sidman, 1994). Desse ponto de vista, o procedimento adotado parece ser muito promissor tanto para a produção de um responder discriminativo, quanto para a produção de classes de estímulos equivalentes.

É bem verdade que algumas questões ficaram por responder. A primeira delas foi levantada também porTyndall et al. (2004), que afirmaram que não podiam dizer se realmente seus participantes formaram classes de estímulos equivalentes ou se simplesmente separavam os estímulos positivos e negativos em grupos/ classes funcionais, escolhendo os estímulos-comparação da mesma classe do estímulo-modelo. O mesmo pode ser dito deste trabalho, especialmente considerando-se os desempenhos dos participantes PLE e N nos testes. No entanto, possivelmente com base nesta partição, pelo menos para alguns participantes emergiram relações condicionais não treinadas entre os estímulos, assim como ocorreu nos estudos de Sidman et al. (1989) e Tyndall et al. (2004).

A segunda questão que merece reflexão e investigação mais aprofundada diz respeito a como exatamente caracterizar o procedimento de sobreposição de estímulos utilizado. Quando os estímulos apresentados tinham fundo colorido (estímulos A) e uma forma desenhada (estímulos B ou C), o participante estava diante de um estímulo composto, o que aproximaria este estudo dos de Mallot et al. (1971), Zental e Hogan (1975), Debert (2003). No entanto, uma das dimensões dos estímulos compostos tinha já função comportamental (de $\mathrm{S}^{+}$ou de $\mathrm{S}$ ), o que destacava uma das dimensões do estímulo de outra e, como mostram os resultados obtidos, com implicações importantes em termos de eficiência do treino e de possibilidade de formação de classes de estímulos. Desse ponto de vista, cada dimensão do estímulo composto poderia ser vista como possuidora de uma função distinta e, talvez, o treino discriminativo envolvendo a sobreposição de estímulos já estabelecidos como S+ ou S sobre estímulos"neutros" poderia ser tomada como um caso especial de emparelhamento de acordo com o modelo, no qual o estímulo com função comportamental seria um estímulo-modelo.

Ainda que se assuma esta última posição - a de que o procedimento de treino discriminativo utilizado envolveu uma forma de discriminação condicional permanece o fato de que pouquíssimas tentativas foram necessárias para se encerrar o treino com sucesso e para a obtenção de resultados positivos nos testes de formação de classes de estímulos equivalentes (para alguns participantes). Este resultado sugere a existência de alguma característica especial no procedimento utilizado: especula-se que, além do pareamento entre estímulos, o estabelecimento prévio de uma função comportamental serviu como importante facilitador no treino e, por consequência, para a eventual formação de classes de estímulos. Diferentes procedimentos e questões de controle de estímulos relacionadas a aspectos específicos de treinos discriminativos e seus efeitos sobre a emergência de classes de equivalência vêm sendo investigados, com resultados que sugerem que muitas e distintas variáveis podem ser relevantes para a formação de classes de estímulos equivalentes (Assis, Baptista, Kato \& Menezes, 2004; Bagaiolo \& Micheletto, 2004; Debert, 2003; Domeniconi, Costa, Souza \& De Rose, 2007; Lima \& Assis, 2003; Lopes Junior \& Costa, 2003; Malott et al., 1971). A hipótese aqui levantada também merece ser testada, e estudos futuros poderiam fazê-lo. Nesta direção, tais pesquisas poderiam investigar os efeitos de variação dos procedimentos de 
sobreposição de estímulos, especialmente os efeitos de sobrepor apenas estímulos com função de $\mathrm{S}^{+}$ou de $S_{\text {; }}$, por exemplo.

Ainda resta por discutir a questão da variabilidade nos resultados dos testes, pois, dado o treino realizado, as mesmas relações entre estímulos não foram estabelecidas, ou ainda as mesmas classes de estímulos não foram formadas para todos os participantes. A variabilidade tem sido comum nos estudos que investigam os efeitos de diferentes treinos sobre a formação de classes de estímulos, Assis et al. (2004), Bagaiolo e Micheletto (2004), Debert (2003), Domeniconi et al. (2007), Lima e Assis (2003), Lopes Junior e Costa (2003), Wirth e Chase (2002) e Sidman et al. (1989) e tem sido difícil de explicar. Para alguns participantes, O"fracasso"nos testes parece ter se devido a um desempenho que envolveu um responder especialmente forte que parecia ser controlado pela exclusão dos estímulos estabelecidos como S; ou dos estímulos associados a esses estímulos. Esta hipótese, que aqui pôde ser avaliada com algum rigor, pode valer em casos de treino de discriminação condicional e testes de formação de classes de estímulos que utilizam procedimento de emparelhamento de acordo com o modelo; também nesses casos, o"fracasso"no teste pode estar ligado a um responder por exclusão diante de estímulos que, no treino, por alguma razão, tenham assumido função comportamental de S-

Também é importante ressaltar que resultados variáveis não têm sido incomuns na literatura sobre controle de estímulos, assim como não tem sido incomum que treino ou testes adicionais promovam a formação de classes de estímulos naqueles participantes que, de início, têm desempenho aquém do esperado. Poder-se-ia aqui também levantar a hipótese de que treino ou testes adicionais teriam algum efeito sobre os resultados daqueles participantes com desempenhos que não indicaram responder sob controle de classes de estímulos equivalentes nos testes realizados.

Finalmente, a maior contribuição deste estudo talvez tenha sido que dele é possível levantar uma questão oposta àquela que até aqui foi debatida e que merece mais investigação: que aspecto do procedimento, ou que outra variável - do participante, da situação - foi tão relevante a ponto de promover o estabelecimento de classes de estímulos e sua ampliação, quase sem treino e sem erros?

\section{Referências}

Assis, G., Baptista, M. G., Kato, O. M., \& Menezes, A. B. (2004). Discriminações condicionais após treino de pareamento consistente de estímulos complexos. Estudos de Psicologia (Natal), 9 (2), 297-308.

Bagaiolo, L. F., \& Micheletto, N. (2004). Fading e exclusão: aquisição de discriminações condicionais e formação de classes de estímulos equivalentes. Temas em Psicologia, $12(1), 168-185$.

Bonito, M. A. (2005). Um procedimento para medir o estabelecimento de controle de estímulos em uma discriminação simples, baseado em Touchette (1971). Dissertação de mestrado não-publicada, Programa de Estudos Pós-Graduados em Psicologia Experimental: Análise do Comportamento, Pontifícia Universidade Católica de São Paulo.

Debert, P. (2003). Relações condicionais com estímulos compostos. Tese de doutorado não-publicada, Instituto de Psicologia, Universidade de São Paulo.

Domeniconi, C., Costa, A. R. A., Souza, D. G., \& De Rose, J. C. (2007). Responder por exclusão em crianças de 2 a 3 anos em uma situação de brincadeira. Psicologia Reflexão e Crítica, 20 (2), 342-350

De Rose, J. C. (1993). Análise comportamental da cognição. Psicologia: Teoria e Pesquisa, 9 (2), 283- 303.

Goldiamond, I. (1966). Perception, language and conceptualization rules. In B. Kleinmuntz (Ed.), Problem solving: research, method, and theory. New York: Wiley.

Jitsumori, M., Siemann, M., Lehr, M., \& Delius, J. D. (2002). A new approach to the formation of equivalence classes in pigeons. Journal of the Experimental Analysis of Behavior, 78 (3), 397-408.

Kastak, C. R., Schusterman, R. J., \& Kastak, D. (2001). Equivalence classification by California sea Lions using class-specific reinforcers. Journal of the Experimental Analysis of Behavior, 76 (2), 131-158.

Lima, M. P., \& Assis, G. (2003). Emergência de classes seqüenciais após treino com pareamento consistente. Psicologia: Teoria e Pesquisa, 19 (1), 75-84.

Lopes Junior, J., \& Costa, G. G. (2003). Efeitos das respostas de observação diferenciais sobre a aprendizagem de relações condicionais com estímulos complexos. Psicologia Reflexão e Crítica, 16 (1), 71-84.

Mallot, R. W., Mallot, K., Svinicki, J. G., Kladder, F., \& Ponicki, E. (1971). An analysis of matching and nonmatching behavior using a single key, free operant procedure. Psychological Record, 21, 545-564.

Sidman, M. (1986). Functional analysis of emergent verbal classes. In T. Thompson \& M. D. Zeiler (Eds.), Analysis and integrations of behavioral units. New Jersey: Lawrence Erlbaum Associates.

Sidman, M. (1994). Equivalence and the three-term unit. In M. Sidman. Equivalence relations and behavior: a research story. Boston: Authors Cooperative. 
Sidman, M. (2000). Equivalence relations and the reinforcement contingency. Journal of the Experimental Analysis of Behavior, 74 (1), 127-146.

Sidman, M., \& Tailby, W. (1982) Conditional discrimination vs. matching to sample: an expansion of the testing paradigm. Journal of the Experimental Analysis of Behavior, $37(1), 5-22$.

Sidman, M., Wynne, C. K., Maguire, R. W., \& Barnes, T. (1989). Functional classes and equivalence relations. Journal of the Experimental Analysis of Behavior, 52 (3), 261-274.

Skinner, B. F. (1959). Cumulative record. New York: AppletonCentury-Crofts.

Tomonaga, M. (1999). Establishing functional classes in a chimpanzee (Pan Troglodytes) with a two-item sequential-responding procedure. Journal of the Experimental Analysis of Behavior, 72 (1), 57-79.

Touchette, P. E. (1971). Transfer of stimulus control: measuring the moment of transfer. Journal of the Experimental Analysis of Behavior, 15 (3), 347- 354.
Tyndall, I. T., Roche, B., \& James, J. E. (2004). The relations between stimulus function and equivalence class formation. Journal of the Experimental Analysis of Behavior, 81 (3), 257-266.

Vaughan Jr, W. (1988). Formation of equivalence sets in pigeons. Journal of Experimental Psychology: Animal Behavior Processes, 14 (1), 36 -42.

Wirth, O., \& Chase, P. N. (2002). Stability of functional equivalence and stimulus equivalence: effects of baseline reversals. Journal of the Experimental Analysis of Behavior, 77 (1), 29-47.

Zentall, T. R., \& Hogan, D. E. (1975). Concept learning in the pigeon: transfer to new matching and nonmatching stimuli. American Journal of Psychology, 88 (2), 233-244.

Recebido em: 8/11/2006

Versão final reapresentada em: 25/4/2008

Aprovado em: 19/5/2008 